\title{
GALEX Constraints on AGN Feedback in Early-Type Galaxies
}

\author{
Sukyoung K. Yi \\ Yonsei University, Department of Astronomy, Seoul 120-749, South Korea \\ Email: yi@yonsei.ac.kr
}

\begin{abstract}
The absence of vigorous star formation in early-type galaxies is a mystery because early-type galaxies do have a copious supply of cold gas. Various heating mechanisms have been put forward to explain this, and AGN feedback is one of the most widely suspected culprits. The GALEX UV telescope detects even a small amount of star formation and allows an estimate of the current and recent star formation. We find that star formation is still common in earlytype galaxies with low velocity dispersion where the black hole mass is believed to be small. We compare the star-formation rates of the galaxies with predictions from various semi-analytic models with different feedback prescriptions. We find that the passive behavior of central galaxies in groups and clusters is reproduced by semi-analytic models with AGN feedback. However, the same prescriptions causes satellite (i.e., non-central) galaxies to be quenched as well, much more than observed by GALEX, leading to a "satellite over-quenching problem."
\end{abstract}

Keywords. galaxies: elliptical and lenticular, cD, galaxies: evolution

\section{Introduction}

As is evident from the title of this conference, the co-evolution of central black holes and their host galaxies has been a hottly debated issue in modern astrophysics. Maggorian et al. (1998) opened up the discussion with the discovery of a tight correlation between black hole masses and bulge masses. Since proper references to the landmark papers in this area are found in other reviews in this volume, I would like to introduce our recent findings mainly based on new UV data made available by the GALEX space telescope. This paper summaries work described in detail by Schawinski et al. (2006), Kimm et al. (2009), and Kimm et al. (2010, in preparation).

\section{Residual Star Formation in Early-Type Galaxies}

The all-sky UV survey performed by GALEX allows us to see what was unseen before. The UV data on apparently elliptical galaxies reveals that a fairly large fraction of earlytype galaxies in the local universe have formed stars in the last billion years (Yi et al. 2005; Kaviraj et al. 2007; Schawinski et al. 2007a; Salim et al. 2007). This fraction is somewhat uncertain, but ranges from 10 to $50 \%$ depending on the details of the analysis. But considering that the UV detects stars formed only in the past billion years or so, it is quite likely that many early-type galaxies have been forming stars in the last several billion years, at least in a residual mode. Typical early-type galaxies seem to have a few tenths of a percent of the stellar mass in a young population about a few hundred million years old.

It is remarkable that, unlike in previous works, a statistically significant sample shows that even super- $L^{*}$ early-type galaxies have been forming stars. The past misconception that early types are dead has proven to be incorrect, while it is true that they are almost dead. 


\section{AGN Feedback}

We find that early-type galaxies with lower velocity dispersions are much more likely to experience residual star formation (Schawinski et al. 2007a). But the correlation is not as tight when we use galaxy luminosity or stellar mass in lieu of velocity dispersion. We pay heed to the fact that black hole mass correlates most tightly with velocity dispersion (e.g., Tremaine et al. 2002). Motivated by this, we performed a semi-analytical simulation with an ad hoc assumption that residual star formation would be quenched if the black hole mass exceeds a certain critical value for a given velocity dispersion (Schawinski et al. 2006). This simulation reproduces the relation between velocity dispersion and UV bright early-type galaxy fraction reasonably well. While this simulation is very simplistic and $a d h o c$, it gives some hint to the likely role of supermassive black holes in the residual star-formation activity in low star-formation environments such as elliptical galaxies.

Such a role is apparent in optical studies as well. We find that early-type galaxies show a very clear transition from star-forming, through transition objects and Seyfert $2 \mathrm{~s}$, to quiescent galaxies (Schawinski et al. 2007b). The sequence seems consistent with a picture where early-type galaxies have a cold gas supply (from various channels), and the cold gas turns into stars, but also gradually reaches the central black hole. The black hole becomes active and heats the gas, eventually quenching star formation. This is a possible scenario of AGN feedback.

Being encouraged by this small "apparent" success, we compared the star-formation rates derived from the UV and optical photometric data to the predictions of various semi-analytic models with various AGN prescriptions (Kimm et al. 2009). We find that up-to-date models reproduce the star-formation properties of early-type galaxies that are at the center of their environments, i.e., central galaxy properties are recovered by the models reasonably well. However, we also find that the same models quench star formation in satellite (i.e., non-central) early-type galaxies as well, causing a serious mismatch with observations. In the real world, smaller early-type galaxies with small velocity dispersions are quite often found to show signs of recent star formation, unlike in the models. We note this "satellite over-quenching problem" as a serious new challenge, especially because most galaxies are satellites rather than central galaxies.

\section{Discussion}

AGN feedback is a popular trick theorists like to play with because it is considered to be powerful. But at the same time and conveniently enough, it is understood so poorly that it allows many twists. I feel that it is fairly likely to have an important impact on star-formation activity in bulge-dominant galaxies. Sadly, however, direct observations on the vicinity of black holes are still too challenging, even with modern telescopes. We can perform only circumstantial empirical studies only on the closest galaxies, those within a few tens of megaparsecs. AGN energetics need to be understood much better to give us a clearer view. Until we acquire much more powerful observing techniques, statistical analyses based on large surveys should remain useful. Fortunately, GALEX has made another new type of survey possible.

This article is based on the work of my group, most notably by Sugata Kaviraj, Kevin Schawinski, and Taysun Kimm. This work was supported by Korea Research Foundation Grants (KRF-C00156, Doyak 20090078756). 


\section{References}

Kaviraj, S., et al. 2007, ApJ, 173, 619

Kimm, T., et al. 2009, MNRAS, 394, 1131

Maggorian, J., et al. 1998, ApJ, 647, 676

Salim, S. et al. 2007, ApJ, 173, 267

Schawinski, K., et al. 2007a, ApJ, 173, 512

Schawinski, K., et al. 2007b, MNRAS, 382, 1415

Tremaine, S., et al. 2002, ApJ, 574, 740

Yi, S. K., et al. 2005, ApJ, 619, L111 